\title{
RESOLUÇÃO DE SISTEMAS FLASH UTILIZANDO AS EQUAÇÕES DE ESTADO PENG-ROBINSON, REDLICH- KWONG E SOAVE-REDLICH-KWONG ATRAVÉS DO MÉTODO DE SUBSTITUIÇÕES SUCESSIVAS
}

\author{
H. C. S. FERNANDES ${ }^{1}$, J. NOGUEIRA ${ }^{2}$, A. C. B. ARAÚJO ${ }^{3}$ \\ ${ }^{123}$ Universidade Federal de Campina Grande, Departamento de Engenharia Química \\ E-mail para contato: hellder@gmail.com
}

\begin{abstract}
RESUMO - Esta pesquisa tem como objetivo desenvolver um pacote de códigos computacionais que possibilitaram a resolução de problemas envolvendo equações de estado (EOS). Entre elas estão o cálculo de propriedades termodinâmicas via equações de Peng-Robinson (EOS-PR), Redlich-Kwong (EOS-RK) e Soava-Redlich-Kwong (EOS-SRK). Foi também implementado um modelo para a resolução de um flash multicomponente, chamado de método das substituições sucessivas e este foi aplicado a uma sistema contendo os hidrocarbonetos propano, n-butano, n-pentano e n-hexano. A validação dos resultados foram feitas com base em uma simulação implementada no software Aspen Plus ${ }^{\circledR}$. Nos cálculos validados foram obtidos desvios inferiores a $1 \%$ para o caso da EOS-PR e inferiores a $3 \%$ no caso da EOS-RK e EOS-SRK, mostrando a boa precisão na utilização dos códigos.
\end{abstract}

\section{INTRODUÇÃO}

As resoluções de equações de estado são de fundamental importância para o estudo termodinâmico na solução de problemas da engenharia química.

Devido ao grau de complexidade apresentado por algumas das equações torna-se necessário o desenvolvimento de ferramentas computacionais capazes de predizer com exatidão a relação existente entre as propriedades, esta tarefa é bastante importante na engenharia química, pois estas equações podem ser utilizadas na avaliação de desempenho, além de serem de fundamental importância para que trabalhos de modelagem e simulação alcancem resultados satisfatórios.

Para se determinar a solução das equações são necessários parâmetros termodinâmicos das substancias em estudo de cada caso e para se validar os resultados será utilizada simulações no software Aspen Plus®. 


\section{FUNDAMENTAÇÃO TEÓRICA}

\subsection{Equações de Estado (EOS)}

A equação de Peng-Robison (EOS-PR) é recomendada para hidrocarbonetos em processamento de gás, refinaria e processos petroquímicos. Segundo Peng e Robinson (1976) o modelo é da seguinte forma:

$$
\begin{aligned}
& p=\frac{R T}{V_{m}-b}-\frac{a}{V_{m}\left(V_{m}+b\right)+b\left(V_{m}-b\right)} \\
& b=\sum_{i} x_{i} b_{i} \\
& a=\sum_{i} \sum_{j} x_{i} x_{j}\left(a_{i} a_{j}\right)^{0.5}\left(1-k_{i j}\right) \\
& a_{i}=\frac{0.42748 R^{2} T_{c}^{2}}{p_{c}} \\
& b_{i}=\frac{0.08662 R T_{c}}{p_{c}} \\
& k_{i j}=k_{i j}^{(1)}+k_{i j}^{(2)} T+\frac{k_{i j}^{(3)}}{T}
\end{aligned}
$$

Onde, $a$ e $b$ são os parâmetros de correção da não idealidade das substâncias, onde $a$ está relacionado com a força de atração intermolecular e $b$ com volume das moléculas. $\mathrm{O}$ parâmetro $k_{i j}$ representa a interação entre as moléculas i e j respectivamente.

A equação de Redlich-Kwong (EOS-RK) que pode ser usado para sistemas à baixa pressão com um bom resultado (máximo de $8 \mathrm{~atm}$ ). Tal condição é explicada pelo fato da equação não possuir um bom comportamento quando a não idealidade da fase vapor é alta. Também não se é indicada para uso cálculo de propriedades em fase líquida. Segundo Redlich e Kwong (1949) o modelo é da seguinte forma:

$$
\begin{aligned}
& p=\frac{R T}{V_{m}-b}-\frac{\frac{a}{T^{0.5}}}{V_{m}\left(V_{m}+b\right)} \\
& b=\sum_{i} x_{i} b_{i} \\
& a=\sqrt{\sum_{i} x_{i} \sqrt{a_{i}}} \\
& a_{i}=0.42748023 \frac{R^{2} T_{c i}^{2.5}}{p_{c i}} \\
& b_{i}=0.08664035 \frac{R T_{c i}}{p_{c i}}
\end{aligned}
$$


Outra equação é a Soave-Redlich-Kwong (EOS-SRK). Seu modelo se adéqua tanto a baixas como a altas pressões e pode ser utilizado para sistemas de hidrocarbonetos e gases leves como $\mathrm{H}_{2} \mathrm{~S} \mathrm{CO}_{2}$ e $\mathrm{N}_{2}$. Um novo parâmetro de interação entre as moléculas é introduzido. Segundo Soave (1972) o modelo é da seguinte forma:

$$
\begin{aligned}
& p=\frac{R T}{V_{m}-b+c}-\frac{a}{\left(V_{m}+c\right)\left(V_{m}+\mathrm{c}+\mathrm{b}\right)} \\
& a=\left(a_{0}+a_{1}\right) \alpha \\
& b=\sum_{i} x_{i} b_{i} \\
& c=\sum_{i} x_{i} c_{i} \\
& a_{0}=\sum_{i} \sum_{j} x_{i} x_{j}\left(a_{i} a_{j}\right)^{0.5}\left(1-k_{i j}\right) \\
& a_{1}=\sum_{i} x_{i}\left(\sum_{j} x_{j}\left(\left(a_{i} a_{j}\right)^{0.5} l_{i j}\right)^{1 / 3}\right)^{3} \\
& \alpha=\left(1+c\left(1-T_{r}^{2}\right)\right)^{2} \\
& a_{i}=\frac{0.427 R^{2} T_{c}^{2}}{p_{c}} \\
& b_{i}=\frac{0.08664 R T_{c}}{p_{c}} \\
& c_{i}=0.48508+1.55171 \omega-0.15613 \omega^{2} \\
& k_{i j}=k_{i j}^{(1)}+k_{i j}^{(2)} T+\frac{k_{i j}{ }^{(3)}}{T} \\
& l_{i j}=l_{i j}^{(1)}+l_{i j}^{(2)} T+\frac{l_{i j}^{(3)}}{T}
\end{aligned}
$$

Nos dois modelos anteriores (EOS-RK e EOS-SRK ) os parâmetros $a$ e $b$ tem o mesmo significado físico dos mesmos parâmetros na equação de EOS-PR. Entretanto um novo parâmetro $c$ é inserido na equação SRK, que é mais uma correção do volume molecular considerando agora o fator acêntrico das moléculas. O parâmetro lij é uma medição da dificuldade física de interação entre as moléculas em virtude de sua geometria.

\subsection{Sistema Flash}

Considerando um sistema em estado estacionário e em estado de equilíbrio como mostrado na Figura 1 a seguir: 


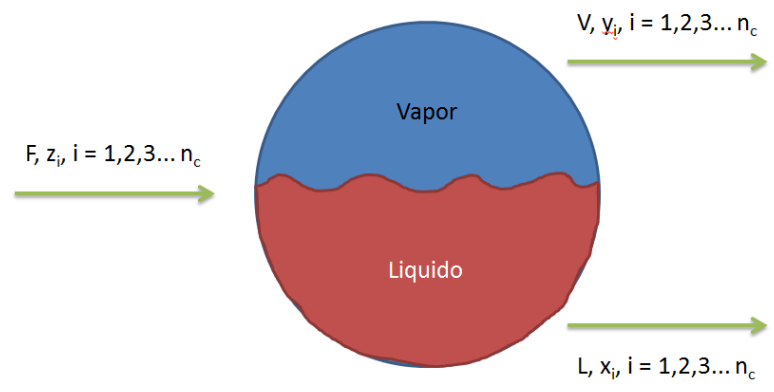

Figura 1 - Estado de equilíbrio entre as fases

Onde $\mathrm{F}$ é a alimentação do sistema com uma composição $\mathrm{z}_{\mathrm{i}}$ ( $\mathrm{i}=$ número de componentes), uma vaporização flash ocorre a uma dada temperatura e pressão ( $\mathrm{T}$ e $\mathrm{P}$ ), originando duas correntes de saída, uma líquida, nomeada de $\mathrm{L}$, como composição $\mathrm{x}_{\mathrm{i}} \mathrm{e}$ uma vapor, $\mathrm{V}$, com composição $\mathrm{y}_{\mathrm{i}}$.

Para um flash, as seguintes equações são resolvidas para se determinar completamente o sistema:

$$
\begin{aligned}
& 0=F-V-L \\
& 0=F z_{i}-V y_{i}-L x_{i} \\
& 0=K_{i} x_{i}-y_{i}
\end{aligned}
$$

As equações acima são respectivamente o balanço de massa geral, o balanço de componentes em ambas as fases e as equações de equilíbrio para cada um dos componentes.

Pode-se rearranjar a equação 26 a fim de se obter uma equação para os termos $\mathrm{x}_{\mathrm{i}}$ e $\mathrm{y}_{\mathrm{i}}$ :

$$
\begin{aligned}
& x_{i}=\frac{z_{i}}{1+\Psi\left(\mathrm{K}_{\mathrm{i}}-1\right)} \\
& y_{i}=\frac{K_{i} z_{i}}{1+\Psi\left(\mathrm{K}_{\mathrm{i}}-1\right)}
\end{aligned}
$$

Onde o termo $\Psi$ corresponde a fração vaporizada V/F. Sabendo que o somatório das frações em ambas as fases é igual a 1:

$$
\sum_{i=1}^{C} y_{i}-\sum_{i=1}^{C} x_{i}=\sum_{i=1}^{C}\left(y_{i}-x_{i}\right)=\sum_{i=1}^{C} \frac{\mathrm{z}_{\mathrm{i}}\left(K_{i}-1\right)}{1+\Psi\left(\mathrm{K}_{\mathrm{i}}-1\right)}=0
$$

A equação 29 é conhecida como equação de Rachford-Rice e é utilizada para a a resolução de um sistema flash com temperatura e pressão especificada. O método de resolução através desta equação é conhecido como método das substituições sucessivas (SSM) e também utiliza a seguinte equação: 


$$
K_{i}=\frac{P_{c i}}{P} \exp \left[5.37\left(1+w_{i}\right)\left(1-\frac{T_{c i}}{T}\right)\right]
$$

A equação 30 é conhecida como a equação de Wilson.

Segundo Naji (2008) para a solução do sistema flash deve-se seguir o seguinte algoritmo apresentado na Figura 2 a seguir:

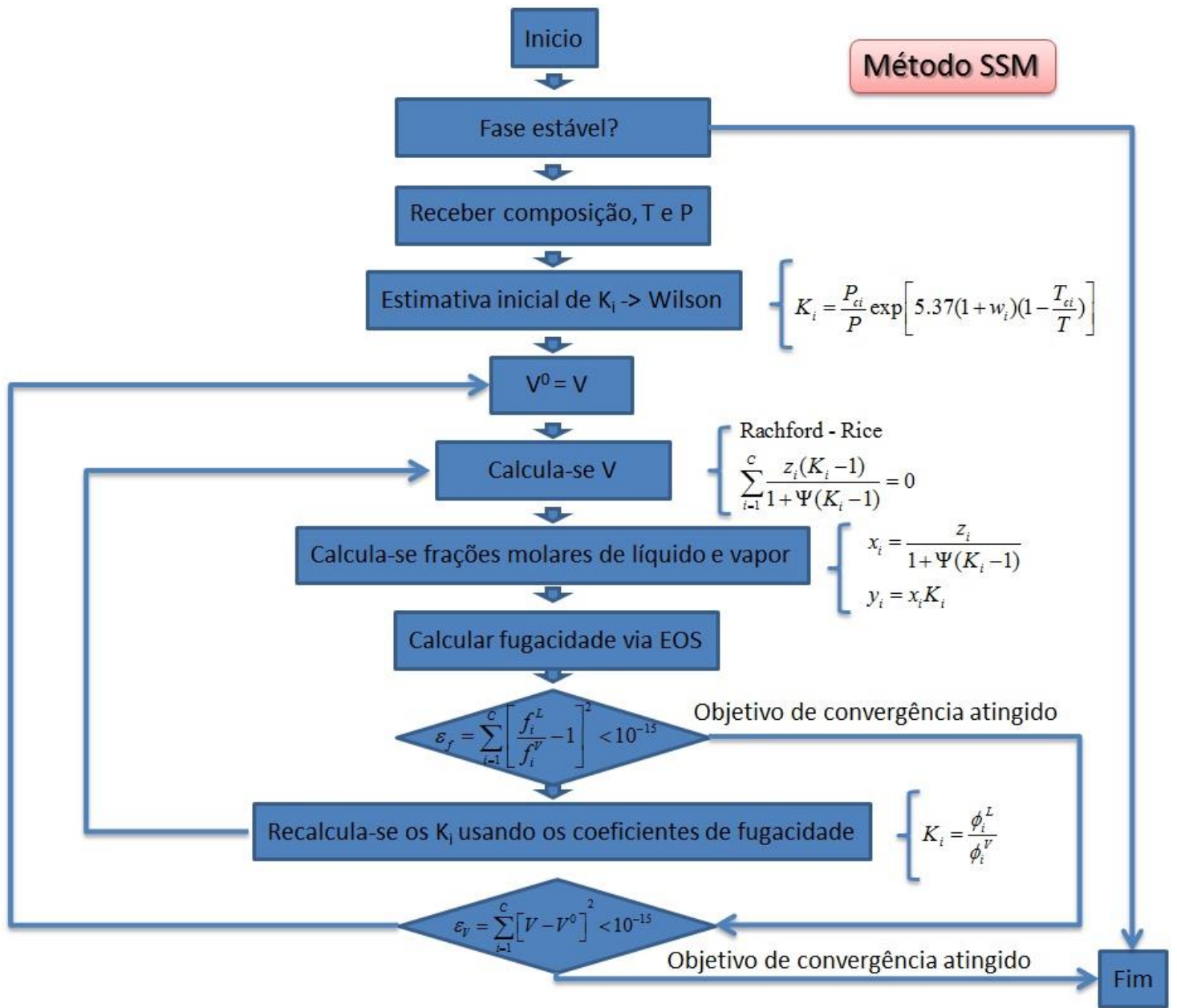

Figura 2 - Algoritmo para resolução de um sistema flash pelo método SSM (fonte: Naji, 2008)

\section{METODOLOGIA}

Para a resolução das equações de estado (RK, SRK e PR) e o método de resolução do flash (SSM), desenvolveu-se um código computacional utilizando as equações dos modelos e aplicou-se em flash que recebe como alimentação uma mistura de propano, n-butano, npentano e n-hexano com as seguintes composições: $0,1,0,2,0,3$ e 0,4. A temperatura e pressão de operação do sistema é de $95^{\circ} \mathrm{C}$ e 7 bar respectivamente. 
Para a validação dos códigos implementou-se a mesma coluna no simulador Aspen Plus ${ }^{\circledR}$ e comparou-se os valores obtidos.

\section{RESULTADOS E DISCUSSÕES}

Primeiramente utilizando-se as equações de estado (EOS) Peng-Robinson, RedlichKwong e Soave-Redlich-Kwong, e o modelo matemático para cálculo do flash (SSM), determinou-se a fração vaporizada da alimentação. Os resultados podem ser observados nas tabela 1 . O desvio (erro relativo) também foi calculado com base na equação 31 a seguir, onde $\mathrm{V}_{\mathrm{e}}$ é o valor dos modelos e $\mathrm{V}_{\mathrm{t}}$ é o valor do simulador Aspen Plus ${ }^{\circledR}$.

$$
\varepsilon(\%)=\frac{\left|V_{e}-V_{t}\right|}{V_{t}} * 100
$$

Tabela 1 - Fração de Vapor calculada para cada EOS

\begin{tabular}{cccr}
\hline Fração de Vapor & $\begin{array}{c}\text { Códigos } \\
\text { implementados }\end{array}$ & $\begin{array}{c}\text { Aspen } \\
\text { Plus }{ }^{\circledR}\end{array}$ & Desvio(\%) \\
\hline PR & 0,16050 & 0,160529 & 0,01807 \\
RK & 0,16190 & 0,164232 & 1,41994 \\
SRK & 0,18430 & 0,193592 & 4,79979 \\
\hline
\end{tabular}

Observa-se que os valores obtidos para a fração de vapor do flash utilizando os três modelos de equação de estado, apresentados na Tabela 1, possuem um erro menor que 5\% quando comparado ao valor do simulador comercial. Sendo o modelo de Peng-Robison o que apresentou o melhor comportamento, o qual o desvio entre os valores foi de aproximadamente $0,018 \%$. A explicação para o desvio mais acentuado nos outros dois modelos está no fato de que o modelo de Redlich-Kwong não apresenta bons resultados para a pressão utilizada, que foi de 7 bar e o próprio simulador comercial faz correções quando o sistema se apresenta em tal condição (correções não aplicadas no código implementado). Já no caso do modelo de Soave-Redlich-Kwong, existe o segundo parâmetro de interação entre as substâncias e este é dificilmente encontrado na literatura, por isso, utilizou-se o valor de zero para estes parâmetros no código desenvolvido, diferentemente do simulador comercial que se utilizou para validar os códigos, o qual possui um banco de dados interno com os valores de tais parâmetros interativos.

Cálculou-se também as composições de cada um dos componentes da mistura em ambas as fases líquida e vapor utilizando as diferentes equações de estado, os valores obtidos estão nas Tabelas 2 à 4.

Tabela 2 - Composições da fase líquida e vapor para a EOS-PR

\begin{tabular}{ccccccc}
\hline & \multicolumn{2}{c}{$\begin{array}{c}\text { Códigos } \\
\text { implementados }\end{array}$} & \multicolumn{2}{c}{ Aspen Plus ${ }^{\circledR}$} & \multicolumn{2}{c}{ Desvio(\%) } \\
\hline Composição & LIQ & VAP & LIQ & VAP & LIQ & VAP \\
Propano & 0,066561 & 0,274861 & 0,066562 & 0,274863 & 0,00150 & 0,00073 \\
N-butano & 0,178339 & 0,313288 & 0,178336 & 0,313289 & 0,00168 & 0,00032 \\
N-Pentano & 0,309620 & 0,249667 & 0,309625 & 0,249669 & 0,00161 & 0,00080 \\
N-Hexano & 0,445470 & 0,162176 & 0,445478 & 0,162179 & 0,00180 & 0,00185 \\
Soma & 1 & 1 & 1 & 1 & & \\
\hline
\end{tabular}


Tabela 3 - Composições da fase líquida e vapor para a EOS-RK

\begin{tabular}{ccccccc}
\hline & \multicolumn{2}{c}{ Códigos } & \multicolumn{2}{c}{ Aspen Plus ${ }^{\circledR}$} & \multicolumn{2}{c}{ Desvio(\%) } \\
& implementados & & & & \\
\hline Composição & LIQ & VAP & LIQ & VAP & LIQ & VAP \\
Propano & 0,068227 & 0,276657 & 0,066102 & 0,272506 & 3,2147 & 1,5232 \\
N-Butano & 0,180680 & 0,320988 & 0,177856 & 0,312687 & 1,5878 & 2,6547 \\
N-Pentano & 0,326892 & 0,26998 & 0,309327 & 0,252536 & 5,6784 & 6,9075 \\
N-Hexano & 0,424202 & 0,152375 & 0,446715 & 0,162271 & 5,0396 & 6,0984 \\
Soma & 1 & 1 & 1 & 1 & & \\
\hline
\end{tabular}

Tabela 4 - Composições da fase líquida e vapor para a EOS-SRK

\begin{tabular}{ccccccc}
\hline & \multicolumn{3}{c}{ Códigos } & \multicolumn{2}{c}{ Aspen Plus ${ }^{\circledR}$} & \multicolumn{2}{c}{ ERRO(\%) } \\
\hline implementados & & & & \\
\hline Composição & LIQ & VAP & LIQ & VAP & LIQ & VAP \\
Propano & 0,06483 & 0,27062 & 0,06355 & 0,25182 & 2,0187 & 7,4659 \\
N-Butano & 0,17653 & 0,31383 & 0,16923 & 0,32813 & 4,3140 & 4,3585 \\
N-Pentano & 0,30993 & 0,25181 & 0,31132 & 0,25284 & 0,4461 & 0,4085 \\
N-Hexano & 0,44869 & 0,16372 & 0,45588 & 0,16719 & 1,5780 & 2,0736 \\
\hline Soma & 1 & 1 & 1 & 1 & & \\
\hline
\end{tabular}

Pelas mesmas razões já explicadas para o caso do cálculo da fração vaporizada, os resultados obtidos com o código implementado da EOS Peng-Robison obtiveram os menores desvios se aproximando muito dos valores do software comercial, onde os mesmos ficaram em valores próximos a $0,001 \%$, mostrando uma boa aplicabilidade da modelagem.

\section{CONCLUSÃO}

Pode-se concluir que a utilização da equação de estado Peng-Robinson junto com o método de substituição sucessiva para a resolução de problemas envolvendo flash tem uma boa aproximação dos valores reais para os hidrocarbonetos estudados (propano, n-butano, npentano e n-hexano), obtendo-se desvios de aproximadamente 0,001 para as composições das fases e $0,01 \%$ para o cálculo de fração vaporizada. Valendo salientar que o método utilizado internamente para a resolução do problema no software comercial é chamado de método inside-out, que é muito mais robusto que o modelo implementado (SSM).

As outras equações de estado implementadas não apresentaram resultados tão bons quando comparado com a de Peng-Robinson principalmente pelas condições do sistema aplicado, no caso da equação de Redlich-Kwong, e pela dificuldade de se encontrar alguns parâmetros das substâncias puras no caso do modelo de Soave-Redlich-Kwong.

\section{REFERÊNCIAS}

Naji, H.S., "Conventional and rapid flash calculations for the Soave-Redlich-Kwong and PengRobinson equations of state", Emirates Journal for Engineering Research, 13 (3), (2008), pp. 81-91. 
PENG, D.Y., ROBINSON, D. B., "A New Two-Constant Equation of state", Ind.Eng. Chem. Fundam., Vol. 15, (1976), pp. 59-64.

REDLICH, O., KWONG J.N.S., "On the Thermodynamics of Solutions V. An Equation of state. Fugacities of Gaseous Solutions”, Chem. Rev., Vol. 44, (1949), pp. $233-244$.

SOAVE, G., "Equilibrium Constants for Modified Redlich-Kwong Equation of state", Chem. Eng. Sci., Vol. 27, (1972), pp. $1196-1203$. 